K. Khashyarmanesh, Sh. Salarian and H. Zakeri

Nagoya Math. J.

Vol. 151 (1998), 37-50

\title{
CHARACTERIZATIONS OF \\ FILTER REGULAR SEQUENCES AND UNCONDITIONED STRONG $d$-SEQUENCES
}

\author{
K. KHASHYARMANESH, SH. SALARIAN AND H. ZAKERI
}

\begin{abstract}
The first part of the paper is concerned, among other things, with a characterization of filter regular sequences in terms of modules of generalized fractions. This characterization leads to a description, in terms of generalized fractions, of the structure of an arbitrary local cohomology module of a finitely generated module over a notherian ring. In the second part of the paper, we establish homomorphisms between the homology modules of a Koszul complex and the homology modules of a certain complex of modules of generalized fractions. Using these homomorphisms, we obtain a characterization of unconditioned strong $d$-sequences.
\end{abstract}

\section{$\S 0$. Introduction}

Throughout this note $A$ is a commutative ring (with non-zero identity), $\mathfrak{a}$ is an ideal of $A$ and $M$ is an $A$-module.

There is a lot of current interest in the theory of filter regular sequences and unconditioned strong $d$-sequences (u.s. $d$-sequences) in commutative algebra; and, in recent years, there have appeared many papers concerned with the role of these sequences in the theory of local cohomology. The main purpose of this note is to establish connections between modules of generalized fractions introduced in [7] and the above mentioned sequences.

This paper is divided in two sections. In the first section we provide a characterization of $\mathfrak{a}$-filter regular sequences in terms of modules of generalized fractions. This result is a slight generalization of the Exactness Theorem [5, 3.1]. In [9], Sharp and the third author proved, in certain situation, for a finitely generated module $N$ over a (Noetherian) local ring $R$ having maximal ideal $\mathfrak{m}$, that the $i$-th local cohomology module $H_{\mathfrak{m}}^{i}(N)$ is isomorphic to the $i$-th homology module of a certain complex of modules of generalized fractions. Our characterization of $\mathfrak{a}$-filter regular sequences, in this paper, yields improved forms of the above theorem and the results

Received May 7, 1996.

Revised April 15, 1997. 
$[1,3.8]$ and $[12,2.5]$ which we mention two in details here. We show, under Noetherian hypothesis on $A$, that

(i) whenever $M$ is finitely generated, then each local cohomology module $H_{\mathfrak{a}}^{i}(M)$ may be viewed as the $i$-th homology module of the complex $C(\mathcal{U}, M)$ of $A$-modules which involves modules of generalized fractions derived from $M$ and an a-filter regular sequence, and that

(ii) whenever $x_{1}, \cdots, x_{n}$ is an a-filter regular sequence on $M$, then

$$
H_{\mathfrak{a}}^{i}(M) \cong H_{\left(x_{1}, \cdots, x_{n}\right)}^{i}(M)
$$

for all $0 \leq i \leq n-1$.

In the second section of this paper, firstly we establish homomorphisms between the homology modules of a Koszul complex and the homology modules of a certain complex of modules of generalized fractions. Then, using these homomorphisms, we obtain a characterization of u.s.d-sequences.

Let us recall briefly the construction of a complex of modules of generalized fractions. The concept of a chain of triangular subsets on $A$ is explained in $\left[5\right.$, p.420]. Such a chain $\mathcal{W}=\left(W_{i}\right)_{i \in \mathbb{N}}$ (Throughout, we use $\mathbb{N}$ to denote the set of positive integers) determines a complex of modules of generalized fractions

$$
0 \stackrel{f^{-1}}{\longrightarrow} M \stackrel{f^{0}}{\longrightarrow} W_{1}^{-1} M \longrightarrow \cdots \longrightarrow W_{i}^{-i} M \stackrel{f^{2}}{\longrightarrow} W_{i+1}^{-i-1} M \longrightarrow \cdots
$$

in which $f^{0}(m)=\frac{m}{(1)}$ for all $m \in M$ and $f^{i}\left(m /\left(w_{1}, \cdots, w_{i}\right)\right)=m /\left(w_{1}, \cdots\right.$, $\left.w_{i}, 1\right)$ for all $i \in \mathbb{N}, m \in M$ and $\left(w_{1}, \cdots, w_{i}\right) \in W_{i}$. We shall denote this complex by $C(\mathcal{W}, M)$.

\section{$\S 1$. A characterization of filter regular sequences}

We say that a sequence $x_{1}, \cdots, x_{n}$ of elements of $A$ is an a-filter regular sequence on $M$ if $x_{1}, \cdots, x_{n} \in \mathfrak{a}$ and

$$
\operatorname{Supp}\left(\left(\left(\sum_{j=1}^{i-1} A x_{j}\right) M:_{M} x_{i}\right) /\left(\sum_{j=1}^{i-1} A x_{j}\right) M\right) \subseteq V(\mathfrak{a})
$$

for all $i=1, \cdots, n$. In particular case, when $A$ is local and $M$ is finitely generated, this notion has been studied in [6], [10], [11] and has led to some interesting results. Note that $x_{1}, \cdots, x_{n}$ is a poor $M$-sequence $[12, \S 2]$ if 
and only if it is an $A$-filter regular sequence on $M$. It is easy to see that the anolouge of $[10$, Appendix, 2 (ii)] is held whenever $A$ is Noetherian, $M$ is finitely generated and $\mathfrak{m}$ is replaced by $\mathfrak{a}$; so that, if $x_{1}, \cdots, x_{n}$ is an $\mathfrak{a}$-filter regular sequence on $M$, then there is an element $y \in \mathfrak{a}$ such that $x_{1}, \cdots, x_{n}, y$ is an $\mathfrak{a}$-filter regular sequence. Thus, for a positive integer $n$, there exists an $\mathfrak{a}$-filter regular sequence on $M$ of length $n$.

Let $I$ be the set of positive integers or $I=\{1, \ldots, n\}$ for some $n \in \mathbb{N}$. In this section we shall work with a family $\mathcal{U}=\left\{U_{i}\right\}_{i \in I}$ of sets which has the following properties:

(i) for all $i \in I, U_{i}$ is a triangular subset of $A^{i}$ such that $U_{i} \subseteq(\mathfrak{a}-\{0\})^{i}$,

(ii) whenever $\left(u_{1}, \ldots, u_{i}\right) \in U_{i}$ with $i>1$, then $\left(u_{1}, \ldots, u_{i-1}\right) \in U_{i-1}$

Given such a family $\mathcal{U}$, set

$$
\begin{array}{r}
\bar{U}_{i}=\left\{\left(y_{1}, \ldots, y_{i}\right): \text { there exists } j \text { with } 0 \leq j \leq i\right. \text { such that } \\
\left.\left(y_{1}, \ldots, y_{j}\right) \in U_{j} \text { and } y_{j+1}=\cdots=y_{i}=1\right\}
\end{array}
$$

for all $i \in \mathbb{N}$. Then $\overline{\mathcal{U}}=\left\{\bar{U}_{i}\right\}_{i \in \mathbb{N}}$ is a chain of triangular subsets on $A$; hence, as we mentioned in the introduction, we may construct the complex $C(\overline{\mathcal{U}}, M)$.

The next theorem, which characterizes the concept of an $\mathfrak{a}$-filter regular sequence in terms of modules of generalized fractions, is an improved form of the general exactness theorem $[5,3.1]$.

THEOREM 1.1. With the above notation, each element of $U_{i}$ is an afilter regular sequence on $M$ for all $i \in I$ if and only if $\operatorname{Supp}\left(H^{j}(C(\overline{\mathcal{U}}, M)) \subseteq\right.$ $V(\mathfrak{a})$ for all $j \in I$, where $H^{j}(C(\overline{\mathcal{U}}, M))$ denotes the $j$-th homology module of $C(\overline{\mathcal{U}}, M)$.

Proof. Write the complex $C(\overline{\mathcal{U}}, M)$ as

$$
0 \longrightarrow M \stackrel{d^{0}}{\longrightarrow} \bar{U}_{1}^{-1} M \stackrel{d^{1}}{\longrightarrow} \cdots \longrightarrow \bar{U}_{i}^{-i} M \stackrel{d^{i}}{\longrightarrow} \bar{U}_{i+1}^{-i-1} M \longrightarrow \cdots
$$

and suppose that, for all $i \in I$, each element of $U_{i}$ is an a-filter regular sequence. Let $P \notin V(\mathfrak{a})$. Let $\varphi: A \longrightarrow A_{P}$ be the natural homomorphism and, for all $i \in \mathbb{N}$, set

$$
\bar{U}_{i P}=\left\{\left(\varphi\left(y_{1}\right), \ldots, \varphi\left(y_{i}\right)\right):\left(y_{1}, \ldots, y_{i}\right) \in \bar{U}_{i}\right\}
$$


which is a triangular subset of $\left(A_{P}\right)^{i}$. Then each element of $\bar{U}_{i P}$ is a poor $M_{P}$-sequence for all $i \in I$. Hence, by $[5,3.1]$, the complex

$$
0 \longrightarrow M_{P} \longrightarrow \bar{U}_{1 P}^{-1} M_{P} \longrightarrow \cdots \longrightarrow \bar{U}_{i-1 P}^{-i+1} M_{P} \longrightarrow \bar{U}_{i p}^{-i} M_{P} \longrightarrow \cdots
$$

is exact. Therefore, in view of $[2,2.1], P \notin \operatorname{Supp}\left(\operatorname{ker} d^{i} / \mathrm{im} d^{i-1}\right)$ for all $i \in I$. This proves one side of the theorem. A similar argument shows that the other side is also true.

For the remaining part of this section, we shall assume that $A$ is Noetherian. One can use the arguments in the proof of $[9,2.4]$ to obtain the following theorem.

THEOREM 1.2. Let $n \in \mathbb{N}$ and let $\mathcal{W}=\left(W_{i}\right)_{i \in \mathbb{N}}$ be a chain of triangular subsets on $A$ such that

(i) for all $i=1, \cdots, n, H_{\mathfrak{a}}^{j}\left(W_{i}^{-i} M\right)=0$ for every $j \in \mathbb{N} \cup\{0\}$, and,

(ii) for all $i=0, \cdots, n-1$, Supp $\left(H^{i}(C(\mathcal{W}, M))\right) \subseteq V(\mathfrak{a})$.

Then $H^{i}(C(\mathcal{W}, M)) \cong H_{\mathfrak{a}}^{i}(M)$ for all $i=0, \cdots, n-1$.

In the next consequences, of the above theorems, we improve the results $[9,2.4],[1,3.8]$ and $[12,2.5]$ which are mentioned in the introduction.

CONSEQUENCES 1.3. (i) Suppose that $M$ is finitely generated and let $I=\{1, \ldots, n\}$. Let $x_{1}, \ldots, x_{n}$ be an a-filter regular sequence on $M$. (Note that the existence of such sequences is explained in the beginning of this section). Let $\mathcal{U}=\left\{U_{i}\right\}_{i \in I}$, where

$$
U_{i}=\left\{\left(x_{1}^{\alpha_{1}}, \ldots, x_{i}^{\alpha_{i}}\right): \alpha_{1}, \ldots, \alpha_{i} \in \mathbb{N}\right\}
$$

for all $i \in I$. With the above notations, since, by $[7,3.2] \bar{U}_{i}^{-i} M \cong U_{i}^{-i} M$ for all $i \in I$, we have, by $[8,2.2]$, that $H_{\mathfrak{a}}^{j}\left(\bar{U}_{i}^{-i} M\right)=0$ for all $j \in \mathbb{N} \bigcup\{0\}$ and $i \in I$. Hence, by 1.1 and $1.2, H^{i}(C(\overline{\mathcal{U}}, M)) \cong H_{\mathfrak{a}}^{i}(M)$ for all $i=0, \ldots$, $n-1$.

(ii) Let $x_{1}, \ldots, x_{n}$ be an a-filter regular sequence on $M$. Since $x_{1}, \ldots, x_{n}$ as well as is an $\sum_{i=1}^{n} A x_{i}$-filter regular sequence on $M$, we deduce, by using the argument as in (i), that $H_{\mathfrak{a}}^{i}(M) \cong H_{\left(x_{1}, \ldots, x_{n}\right)}^{i}(M)$ for all $i=0,1, \ldots$, $n-1$. 


\section{$\S 2$. A characterization of unconditioned strong $d$-sequences}

For a system of elements $x=x_{1}, \cdots, x_{n}$ of $A$, let $K_{\bullet}(x, A)$ and $H_{p}(x, A)$ denote the Koszul complex generated by $x$ over $A$ and the $p$-th homology module of Koszul complex, respectively. When discussing Koszul complex, we shall use the notations of [4]. In particular, we shall abbreviate $K_{p}(x, A)$ to $K_{p}$ when no confusion is possible. For each $i \in \mathbb{N}$ set

$$
\begin{aligned}
U(x)_{i}=\left\{\left(x_{1}^{\alpha_{1}}, \cdots, x_{i}^{\alpha_{i}}\right): \text { there exists } j \text { with } 0 \leq j \leq i\right. \\
\text { such that } \left.\alpha_{1}, \cdots, \alpha_{j} \in \mathbb{N} \text { and } \alpha_{j+1}=\cdots=\alpha_{i}=0\right\},
\end{aligned}
$$

where $x_{r}$ is interpreted as 1 whenever $r>n$. Then, for each $i \in \mathbb{N}, U(x)_{i}$ is a triangular subset of $A^{i}$. We use $\mathcal{A}(x)$ to denote the family $\left(U(x)_{i}\right)_{i \in \mathbb{N}}$. Write the associated complex $C(\mathcal{A}(x), A)$ as

$$
0 \stackrel{e_{x, A}^{-1}}{\rightarrow} A \stackrel{e_{x, A}^{0}}{\rightarrow} U(x)_{1}^{-1} A \rightarrow \cdots \rightarrow U(x)_{i}^{-i} A \stackrel{e_{x, A}^{i}}{\rightarrow} U(x)_{i+1}^{-i-1} A \rightarrow \cdots .
$$

It will be convenient to allow $U(x)_{0}^{-0} A$ and $U(x)_{-1}^{-(-1)} A$ to denote $A$ and 0 respectively.

Next, in order to characterize the concept of an u.s. $d$-sequence, we establish homomorphisms from the homology modules of the complex $K_{\bullet}(x, A)$ to the homology modules of the complex $C(\mathcal{A}(x), A)$, and we consider the commutative diagrams which are needed in the proof of the characterization. For each integer $p$ with $0 \leq p \leq n$, we define $\Psi_{x, A}^{p}: K_{n-p} \longrightarrow$ $U(x)_{p}^{-p} A$ as follows. $\Psi_{x, A}^{0}: K_{n} \longrightarrow A$ is the identity map, $\Psi_{x, A}^{n}: K_{0} \longrightarrow$ $U(x)_{n}^{-n} A$ is such that $\Psi_{x, A}^{n}(1)=\frac{1}{\left(x_{1}, \cdots, x_{n}\right)}$ and, for each $1 \leq p \leq n-1$, $\Psi_{x, A}^{p}: K_{n-p} \longrightarrow U(x)_{p}^{-p} A$ is defined by the rule

$$
\Psi_{x, A}^{p}\left(e_{i_{1} \cdots i_{n-p}}\right)= \begin{cases}\frac{1}{\left(x_{1}, \cdots, x_{p}\right)} & \text { if }\left(i_{1}, \cdots, i_{n-p}\right)=(p+1, \cdots, n) \\ 0 & \text { otherwise. }\end{cases}
$$

It is easily seen that, for all $0 \leq p \leq n . \Psi_{x, A}^{p}$ is a well-defined $A$-homomorphism and that the diagram

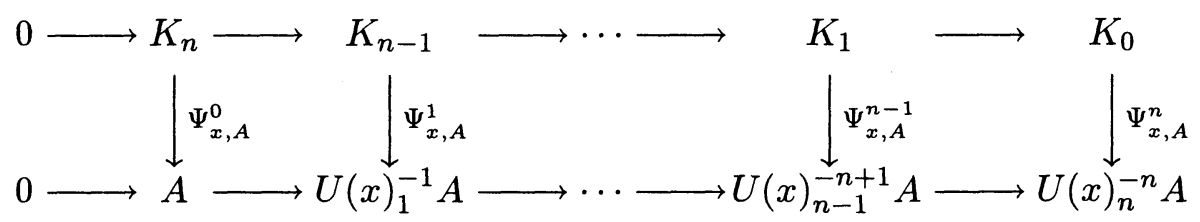


is commutative. Therefore, for all $0 \leq p \leq n-1, \Psi_{x, A}^{p}$ induces an $A$ homomorphism $H_{n-p}(x, A) \longrightarrow \frac{\operatorname{ker} e_{x, A}^{p}}{\operatorname{im} e_{x, A}^{p-1}}$ which we denote by $\bar{\Psi}_{x, A}^{p}$. Let $n \geq 2$ and set $\underline{x}=x_{1}, \cdots, x_{n-1}$. Let $K_{\bullet}^{\prime}(\underline{x}, A)$ be the complex obtained from $K_{\bullet}(\underline{x}, A)$ by shifting the degrees up by 1 (that is $K_{p+1}^{\prime}(\underline{x}, A)=K_{p}(\underline{x}, A)$ and the differential of $K_{\bullet}^{\prime}(\underline{x}, A)$ is that of $\left.K_{\bullet}(\underline{x}, A)\right)$. Then there is a morphism of complexes

$$
(g)^{\bullet}=\left(g_{p}\right)_{p}: K_{\bullet}\left(\underline{x}, x_{n}, A\right) \longrightarrow K_{\bullet}^{\prime}(\underline{x}, A)
$$

which is such that, for all $p \in\{1, \ldots, n\}$ the $A$-homomorphism $g_{p}: K_{p} \longrightarrow$ $K_{p}^{\prime}$ is defined by the rule

$$
g_{p}\left(e_{i_{1} \cdots i_{p}}\right)= \begin{cases}e_{i_{1} \cdots i_{p-1}} & \text { if } i_{p}=n \\ 0 & \text { otherwise }\end{cases}
$$

Also, with the above notations, it is straight forward to check that, for all $-1 \leq i \leq n-2$, the diagram

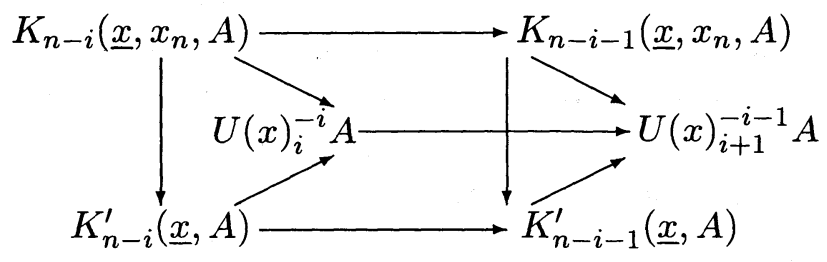

commutes. Thus, for all $0 \leq i \leq n-2$, there is a commutative diagram

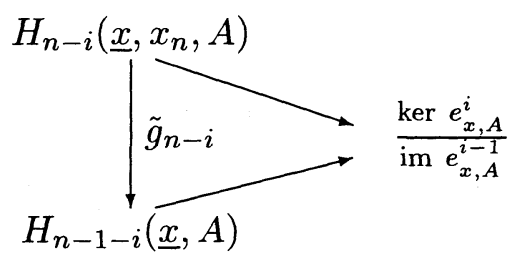

in which the homomorphism $\tilde{g}_{n-i}$ is induced by $(g)^{\bullet}$.

Remark 2.1. Consider for the moment the special case in which $A$ is Noetherian and $\bar{\Psi}_{x, A}^{p}$ is surjective for all $0 \leq p \leq n-1$. Then the hypotheses 
of Theorem 1.2 are satisfied and therefore $H_{\left(x_{1}, \cdots, x_{n}\right)}^{p}(A) \cong \frac{\operatorname{ker} e_{x, A}^{p}}{\operatorname{im} e_{x, A}^{p-1}}$ for all $p=0, \cdots, n-1$. If moreover $A$ is a local ring and $x_{1}, \cdots, x_{n}$ is a system of parameters for $A$, then $A$ is generalized Cohen-Macaulay local ring.

The theory of $d$-sequences was introduced by Huneke in [3]. The sequence $x_{1}, \cdots, x_{n}$ of elements of $A$ is called a $d$-sequence on $M$ if, for each $i=0, \cdots, n-1$, the equality

$$
\left(\sum_{j=1}^{i} A x_{j}\right) M:_{M} x_{i+1} x_{k}=\left(\sum_{j=1}^{i} A x_{j}\right) M:_{M} x_{k}
$$

hold for all $k \geq i+1$ (this is actually a slight weakening of Huneke's definition); it is an unconditioned strong $d$-sequence (u.s. $d$-sequence) on $M$ if $x_{1}^{\alpha_{1}}, \cdots, x_{n}^{\alpha_{n}}$ is a $d$-sequence in any order for all positive integers $\alpha_{1}, \cdots, \alpha_{n}$. $D$-sequences are closely related to filter regular sequences. It is easy to see that if $x_{1}, \ldots, x_{n}$ is a $d$-sequence on $M$, then it is an $\sum_{i=1}^{n} A x_{i}$-filter regular sequence on $M$. Also, when $A$ is Noetherian and $M$ is finitely generated, by slight modification in the arguments of $[11,2.1]$, one can show that if $x_{1}, \ldots, x_{n}$ is an $\mathfrak{a}$-filter regular sequence on $M$, then, for each $k \geq 0$, there exists an ascending sequence of integers $k \leq r_{1} \leq \ldots \leq r_{n}$ such that $x_{1}^{r_{1}}, \ldots, x_{n}^{r_{n}}$ is a $d$-sequence on $M$. We now gather together the well known properties of $d$-sequences which are needed in this paper.

Remark 2.2. Let $x_{1}, \cdots, x_{n}$ be a $d$-sequence on $M$ and let $y_{1}, \ldots, y_{n}$ be any permutation of $x_{1}, \ldots, x_{n}$. Then:

(i) $x_{1}, \cdots, x_{n}$ form a $d$-sequence on $\frac{M}{\left(0: M x_{1}\right)}$ too. In particular, $x_{1}$ is a non-zero divisor on $\frac{M}{\left(0:_{M} x_{1}\right)}$.

(ii) The sequence $x_{2}, \cdots, x_{n}$ form a $d$-sequence on $\frac{M}{x_{1} M}$.

(iii) If any permutation of $x_{1}, \ldots, x_{n}$ is a $d$-sequence on $M$, then, for each $i=0, \ldots, n-1$, the equality

$$
\left(\sum_{r=1}^{i} A y_{r}\right) M:_{M} y_{j}=\left(\sum_{r=1}^{i} A y_{r}\right):_{M} y_{k}
$$

holds for all $j, k \geq i+1$. 
(iv) If any permutation of $x_{1}, \ldots, x_{n}$ is a $d$-sequence on $M$, then

$$
\left(0:_{M} y_{i}\right) \cap\left(\sum_{r=1}^{n} A y_{r}\right) M=0
$$

for all $i=1, \ldots, n$.

Notations. Let $x=x_{1}, \cdots, x_{n}$ be a sequence of elements of $A$. We put $\bar{A}=\frac{A}{\left(0:_{A} x_{n}\right)}$ and $A^{\prime}=\frac{A}{x_{n} A}$. The natural image of an element $a$ of $A$ in $\bar{A}$ (respectively $A^{\prime}$ ) is denoted by $\bar{a}$ (respectively $a^{\prime}$ ).

There are basic exact sequences which we will use to prove the main theorem in this section. We separate these exact sequences in the following.

LemMA 2.3. Assume that $x=x_{1}, \cdots, x_{n}$ is an u.s.d-sequence on $A$. Then, for each $0 \leq i \leq n$, the sequence

$$
0 \longrightarrow U(x)_{i}^{-i} \bar{A} \stackrel{x_{n}}{\longrightarrow} U(x)_{i}^{-i} A \longrightarrow U(x)_{i}^{-i} A^{\prime} \longrightarrow 0
$$

is exact, where $U(x)_{i}^{-i} A \longrightarrow U(x)_{i}^{-i} A^{\prime}$ is the natural homomorphism.

Proof. It is trivial in case $i=0$. Now suppose that $1 \leq i \leq n$. In view of $[7,2.9]$, the only non-trivial point is the proof that $U(x)_{i}^{-i} \bar{A} \stackrel{x_{n}}{\longrightarrow} U(x)_{i}^{-i} A$ is injective: to do this, let $\frac{x_{n} b}{\left(x_{1}^{\alpha_{1}}, \cdots, x_{i}^{\alpha_{i}}\right)}=0$ in $U(x)_{i}^{-i} A$ for some $b \in A$ and $\left(x_{1}^{\alpha_{1}}, \cdots, x_{i}^{\alpha_{i}}\right) \in U(x)_{i}$. Then, by $[5,1.7]$, there exists $\delta \in \mathbb{N}$ with $\delta \geq \max \left\{\alpha_{1}, \cdots, \alpha_{i}\right\}$ such that

$$
x_{1}^{\delta-\alpha_{1}} \cdots x_{i}^{\delta-\alpha_{i}} x_{n} b \in\left(x_{1}^{\delta}, \cdots, x_{i-1}^{\delta}\right) A .
$$

Since $x_{1}, \cdots, x_{n}$ form an u.s. $d$-sequence on $A$, it follows, by 2.2 (iii), that

$$
x_{1}^{\delta-\alpha_{1}} \cdots x_{i}^{\delta-\alpha_{i}+1} b \in\left(x_{1}^{\delta}, \cdots, x_{i-1}^{\delta}\right) A
$$

Hence $\frac{x_{i} \bar{b}}{\left(x_{1}^{\alpha_{1}}, \cdots, x_{i}^{\alpha_{i}}\right)}=0$ in $U(x)_{i}^{-i} \bar{A}$. Therefore, by $[8,2.1], \frac{\bar{b}}{\left(x_{1}^{\alpha_{1}}, \cdots, x_{i}^{\alpha_{i}}\right)}=0$ in $U(x)_{i}^{-i} \bar{A}$, as required.

Let $x=x_{1}, \cdots, x_{n}$ be an u.s. $d$-sequence on $A$. Consider the complexes $C(\mathcal{A}(x), \bar{A}), C(\mathcal{A}(x), A)$ and $C\left(\mathcal{A}(x), A^{\prime}\right)$. By 2.3 , we can build commuta- 
tive diagram

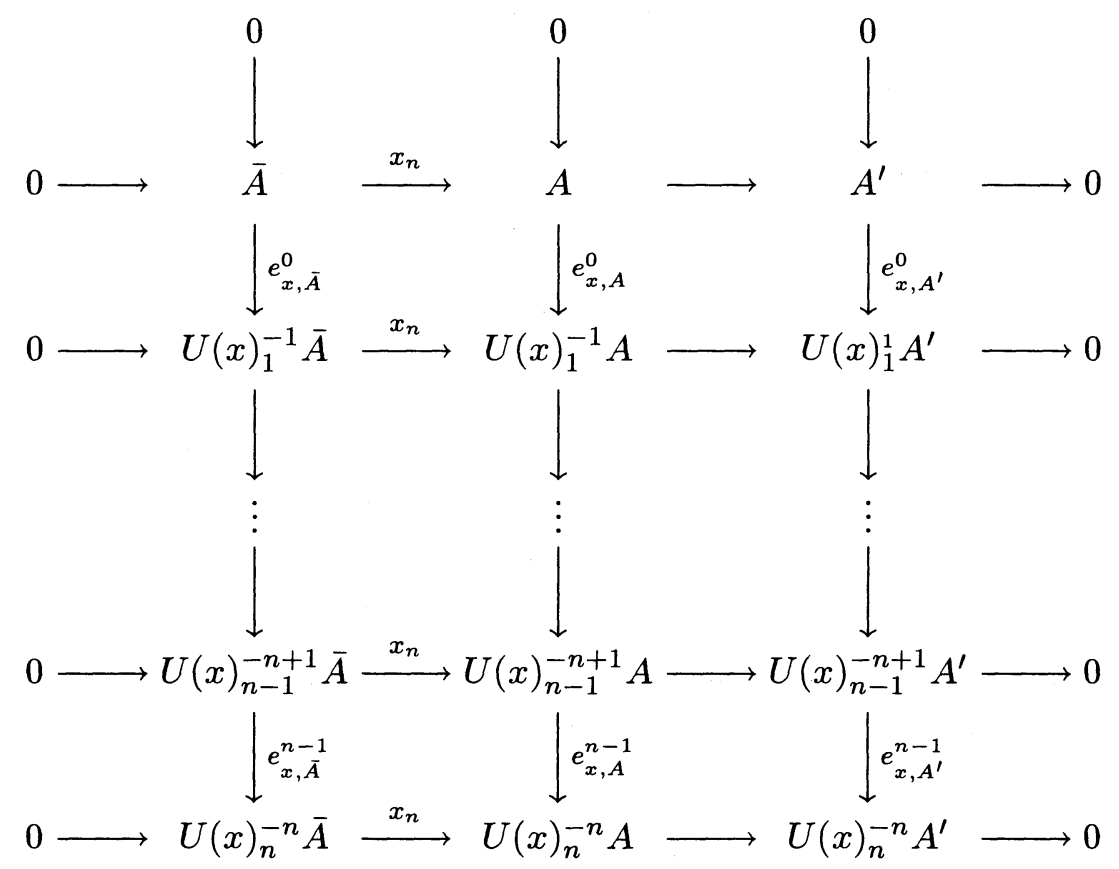

with exact rows which makes the induced exact sequence

$$
\begin{aligned}
0 \longrightarrow \frac{\operatorname{ker} e_{x, \bar{A}}^{0}}{\operatorname{im} e_{x, \bar{A}}^{-1}} \longrightarrow & \frac{\operatorname{ker} e_{x, A}^{0}}{\operatorname{im} e_{x, A}^{-1}} \longrightarrow \frac{\operatorname{ker} e_{x, A^{\prime}}^{0}}{\operatorname{im} e_{x, A^{\prime}}^{-1}} \stackrel{\Delta_{0}}{\longrightarrow} \frac{\operatorname{ker} e_{x, \bar{A}}^{1}}{\operatorname{im} e_{x, \bar{A}}^{0}} \longrightarrow \cdots \\
& \stackrel{\Delta_{n-2}}{\longrightarrow} \frac{\operatorname{ker} e_{x, \bar{A}}^{n-1}}{\operatorname{im} e_{x, \bar{A}}^{n-2}} \longrightarrow \frac{\operatorname{ker} e_{x, A}^{n-1}}{\operatorname{im} e_{x, A}^{n-2}} \longrightarrow \frac{\operatorname{ker} e_{x, A^{\prime}}^{n-1}}{\operatorname{im} e_{x, A^{\prime}}^{n-2}}
\end{aligned}
$$

Moreover, we obtain a commutative square

$$
\begin{aligned}
& H_{n-p}\left(x, A^{\prime}\right) \longrightarrow H_{n-p-1}(x, \bar{A})
\end{aligned}
$$

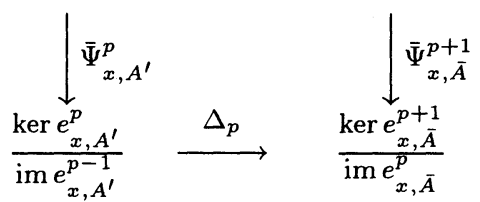

for all $p=0, \ldots, n-2$. Next we show that $\Delta_{p}$ is surjective for all $p=$ $0, \ldots, n-2$. Let $0 \leq p \leq n-2$ and let

$$
\frac{\bar{a}}{\left(x_{1}^{\alpha_{1}}, \cdots, x_{p+1}^{\alpha_{p+1}}\right)} \in \operatorname{ker} e_{x, \bar{A}}^{p+1}
$$


for some $a \in A$ and $\left(x_{1}^{\alpha_{1}}, \cdots, x_{p+1}^{\alpha_{p+1}}\right) \in U(x)_{p+1}$. Then, by $[5,1.7]$ there exists $\delta \in \mathbb{N}$ with $\delta \geq \max \left\{\alpha_{1}, \cdots, \alpha_{p+1}\right\}$ such that

$$
x_{1}^{\delta-\alpha_{1}} \cdots x_{p+1}^{\delta-\alpha_{p+1}} x_{p+2}^{\delta} \bar{a} \in\left(x_{1}^{\delta}, \cdots, x_{p+1}^{\delta}\right) \bar{A} .
$$

Since $x_{1}, \cdots, x_{n}$ is an u.s. $d$-sequence on $A$, we have, by 2.2 (iii), that

$$
x_{1}^{\delta-\alpha_{1}} \cdots x_{p+1}^{\delta-\alpha_{p+1}} x_{n} a \in\left(x_{1}^{\delta}, \cdots, x_{p+1}^{\delta}\right) A .
$$

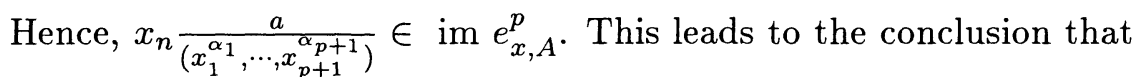

$$
\Delta_{p}: \frac{\operatorname{ker} e_{x, A^{\prime}}^{p}}{\operatorname{im} e_{x, A^{\prime}}^{p-1}} \longrightarrow \frac{\operatorname{ker} e_{x, \bar{A}}^{p+1}}{\operatorname{im} e_{x, \bar{A}}^{p}}
$$

is surjective.

We now come to the main theorem of this section.

THEOREM 2.4. Let $x=x_{1}, \cdots, x_{n}$ be a sequence of elements of $A$. Then the following conditions are equivalent.

(i) $x_{1}, \cdots, x_{n}$ is an u.s.d-sequence on $A$,

(ii) For any permutation $\sigma$ of the set $\{1, \cdots, n\}$, the A-homomorphism

$$
\bar{\Psi}_{\sigma(x), A}^{p}: H_{n-p}(\sigma(x), A) \longrightarrow \frac{\operatorname{ker} e_{\sigma(x), A}^{p}}{\operatorname{im} e_{\sigma(x), A}^{p-1}}
$$

is surjective for all $p$ with $0 \leq p \leq n-1$, where $\sigma(x)=x_{\sigma(1)}, \cdots, x_{\sigma(n)}$.

Proof. (i) $\Longrightarrow$ (ii) We may assume without loss of generality that $\sigma$ is the identity permutation. We prove this by induction on $n$. To begin, note that in the case when $n=1$ is easy. Now suppose inductively that $n>1$ and the result has been proved for smaller value of $n$. Since $x^{\prime}=x_{1}^{\prime}, \cdots, x_{n-1}^{\prime}$ is a u.s. $d$-sequence on $A^{\prime}$, it immediately follows from inductive hypotheses that $\bar{\Psi}_{x^{\prime}, A^{\prime}}^{p}$ is surjective for all integer $p$ with $0 \leq p \leq n-2$. Now, using elementary facts on Koszul complexes and modules of generalized fractions, we obtain the commutative diagram

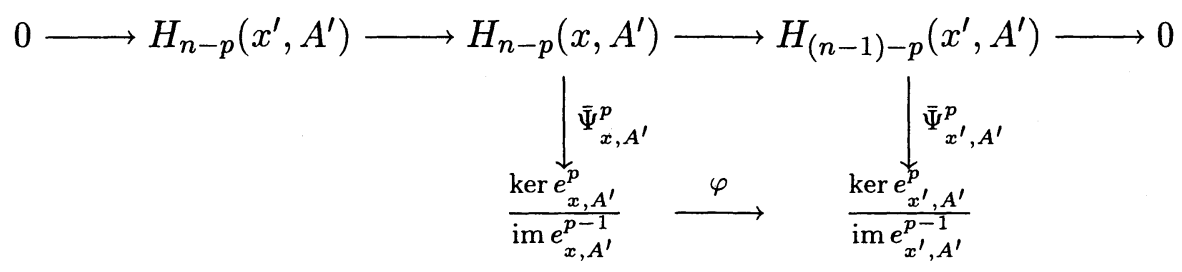


in which the upper row is exact and $\varphi$ is the natural isomorphism. Hence $\bar{\Psi}_{x, A^{\prime}}^{p}$ is surjective for all $p$ with $0 \leq p \leq n-2$. Next, using the exact sequence

$$
0 \longrightarrow\left(0: A x_{n}\right) \longrightarrow A \longrightarrow \bar{A} \longrightarrow 0
$$

we obtain the commutative diagram

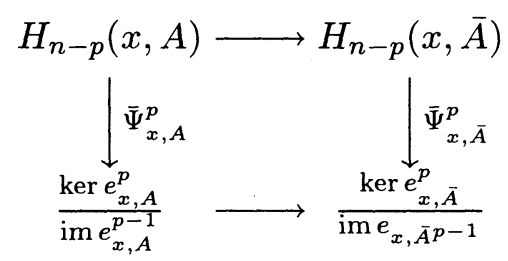

in which, for all $p>0$, the lower row is the natural isomorphism and, since the map $H_{n-p-1}\left(x, 0:_{A} x_{n}\right) \longrightarrow H_{n-p-1}(x, A)$ is injective in view of 2.2 (iv) and the definition of Koszul complex, the upper row is an epimorphism. It now follows, on use of (2), that $\bar{\Psi}_{x, A}^{p}$ is surjective for all $p$ with $0<p \leq n-2$. Finally, since $0:{ }_{A} x_{1}=0:_{A}\left(x_{1}, \ldots, x_{n}\right)$ in view of $2.2($ iii), it is clear that $\bar{\Psi}_{x, A}^{0}$ is the identity map. The result now follows by induction.

(ii) $\Longrightarrow$ (i). We use induction on $n$, the length of the sequence. It is easy in case $n=1$. Now suppose that $n \geq 2$ and assume that our assertion holds for smaller value of $n$. It immediately follows, from the inductive hypothesis and commutative diagram (1), that every sequence of elements of the set $\left\{x_{1}, \cdots, x_{n}\right\}$ of length $n-1$ is u.s. $d$-sequence. Let $\sigma$ be any permutation of the set $\{1, \cdots, n\}$ and let $\alpha_{1}, \cdots, \alpha_{n} \in \mathbb{N}$. In order to prove the result, it suffices to show that

$$
\begin{aligned}
\left(x_{\sigma(1)}^{\alpha_{1}}, \cdots\right. & \left., x_{\sigma(j)}^{v^{\alpha_{j}}}, \cdots, x_{\sigma(n-1)}^{\alpha_{n-1}}\right): x_{\sigma(j)} x_{\sigma(n)} \\
& =\left(x_{\sigma(1)}^{\alpha_{1}}, \cdots, x_{\sigma(j)}^{\alpha_{j}}, \cdots, x_{\sigma(n-1)}^{\alpha_{n-1}}\right): x_{\sigma(n)}
\end{aligned}
$$

for all $j=1, \ldots, n-1$, and

$$
\left(x_{\sigma(1)}^{\alpha_{1}}, \cdots, x_{\sigma(n-1)}^{\alpha_{n-1}}\right): x_{\sigma(n)}^{\alpha_{n}}=\left(x_{\sigma(1)}^{\alpha_{1}}, \cdots, x_{\sigma(n-1)}^{\alpha_{n-1}}\right): x_{\sigma(n)},
$$

where the character with ${ }^{\vee}$ means that it is deleted. We may assume without loss of generality that $\sigma$ is the identity permutation. First we will prove that

$$
\left(x_{1}^{\alpha_{1}}, \cdots,{\stackrel{\vee}{x_{j}}}_{j}^{\alpha_{j}}, \cdots, x_{n-1}^{\alpha_{n-1}}\right): x_{j} x_{n}=\left(x_{1}^{\alpha_{1}}, \cdots, \stackrel{\vee^{\alpha_{j}}}{x_{j}}, \cdots, x_{n-1}^{\alpha_{n-1}}\right): x_{n}
$$


In view of the hypotheses, it suffices to show that

$$
\left(x_{1}^{\alpha_{1}}, \cdots, x_{n-2}^{\alpha_{n-2}}\right): x_{n-1} x_{n}=\left(x_{1}^{\alpha_{1}}, \cdots, x_{n-2}^{\alpha_{n}-2}\right): x_{n} .
$$

To do this, let $r \in\left(x_{1}^{\alpha_{1}}, \cdots, x_{n-2}^{\alpha_{n-2}}\right): x_{n-1} x_{n}$. Then $\frac{r x_{n}}{\left(x_{1}^{\alpha_{1}}, \cdots, x_{n-2}^{\alpha_{n-2}}\right)} \in \operatorname{ker} e_{x, A}^{n-2}$.

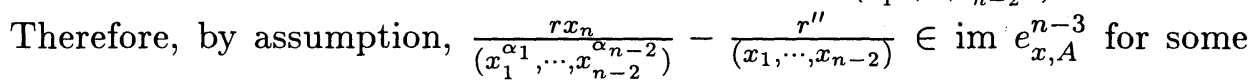
$r^{\prime \prime} \in A$. Consequently, in view of $[5,1.7]$, we have that

$$
x_{1}^{\delta-\alpha_{1}} \cdots x_{n-2}^{\delta-\alpha_{n-2}}\left(r x_{n}-r^{\prime \prime} x_{1}^{\alpha_{1}-1} \cdots x_{n-2}^{\alpha_{n-2}-1}\right) \in\left(x_{1}^{\delta}, \cdots, x_{n-2}^{\delta}\right) A
$$

for some (sufficiently large) integer $\delta$. Hence by $[1,2.3]$

$$
\begin{aligned}
r x_{n}-r^{\prime \prime} & x_{1}^{\alpha_{1}-1} \cdots x_{n-2}^{\alpha_{n-2-1}} \\
& \in \sum_{k=1}^{n-2}\left(x_{1}^{\alpha_{1}}, \cdots, x_{k}^{\alpha_{k}}, \cdots, x_{n-2}^{\alpha_{n-2}}\right): x_{k}^{\alpha_{k}}+\left(x_{1}^{\alpha_{1}}, \cdots, x_{n-2}^{\alpha_{n-2}}\right),
\end{aligned}
$$

because $x_{1}, \cdots, x_{n-2}$ is an u.s. $d$-sequence. Since $x_{1}, \cdots, x_{k}, \cdots, x_{n-2}, x_{n}$ is an u.s. $d$-sequence, we have, by 2.2 (iii), that

$$
\left(x_{1}^{\alpha_{1}}, \cdots, \stackrel{\vee^{\alpha_{k}}}{x_{k}}, \cdots, x_{n-2}^{\alpha_{n-2}}\right): x_{k}^{\alpha_{k}}=\left(x_{1}^{\alpha_{1}}, \cdots, \stackrel{\vee^{\alpha_{k}}}{x_{k}}, \cdots, x_{n-2}^{\alpha_{n-2}}\right): x_{n} .
$$

Also, it is easy to deduce from the definition of the map $\bar{\Psi}_{x, A}^{n}$ and the construction of Koszul complex that

$$
r^{\prime \prime} \in\left(x_{1}, \cdots, x_{n-2}\right): x_{n} .
$$

It therefore follows that

$$
r \in\left(x_{1}^{\alpha_{1}}, \cdots, x_{n-2}^{\alpha_{n-2}}\right): x_{n}^{2}=\left(x_{1}^{\alpha_{1}}, \cdots, x_{n-2}^{\alpha_{n-2}}\right): x_{n}
$$

and this completes the proof of (3).

Next, we will prove that $\left(x_{1}^{\alpha_{1}}, \cdots, x_{n-1}^{\alpha_{n-1}}\right): x_{n}^{\alpha_{n}}=\left(x_{1}^{\alpha_{1}}, \cdots, x_{n-1}^{\alpha_{n-1}}\right): x_{n}$. Let $s \in\left(x_{1}^{\alpha_{1}}, \cdots, x_{n-1}^{\alpha_{n-1}}\right): x_{n}^{\alpha_{n}}$. Then $\frac{s}{\left(x_{1}^{\alpha_{1}}, \cdots, x_{n-1}^{\alpha_{n-1}}\right)} \in \operatorname{ker} e_{x, A}^{n-1}$. Hence, by the same argument as in the proof $(3)$, there exists $s^{\prime \prime} \in\left(x_{1}, \cdots, x_{n-1}\right): x_{n}$ such that

$$
\begin{aligned}
& s-s^{\prime \prime} x_{1}^{\alpha_{1}-1} \cdots x_{n-1}^{\alpha_{n-1}-1} \\
& \quad \in \sum_{k=1}^{n-1}\left(x_{1}^{\alpha_{1}}, \cdots, x_{k}^{\alpha_{k}}, \cdots, x_{n-1}^{\alpha_{n-1}}\right): x_{k}^{\alpha_{k}}+\left(x_{1}^{\alpha_{1}}, \cdots, x_{n-1}^{\alpha_{n-1}}\right) .
\end{aligned}
$$


Now, applying (3), we may deduce that $s \in\left(x_{1}^{\alpha_{1}}, \cdots, x_{n-1}^{\alpha_{n-1}}\right): x_{n}$. The result now follows by induction.

As the referee suggested, one can define the canonical homomorphism $\Psi_{x, M}^{\bullet}$ for every $A$-module $M$ and, by using the same arguments as above, establish 2.4 for modules. Thus 2.4 provides a characterization of Buchsbaum modules in terms of modules of generalized fractions.

Acknowledgements. The authors are deeply grateful to the referee for his careful reading of the manuscript and helpful suggestions. Also the authors would like to thank the Institute for Studies in Theoretical Physics and Mathematics for the financial support.

\section{REFERENCES}

[1] S. Goto and K. Yamagishi, The theory of unconditioned strong d-sequences and modules of finite local cohomology, preprint.

[2] M. A. Hamieh and R. Y. Sharp, Krull dimension and generalized fractions, Proc. Edinburgh Math. Soc., 28 (1985), 349-353.

[3] C. Huneke, The theory of d-sequences and power of ideals, Adv. in Math., 46 (1982), 249-279.

[4] H. Matsumura, Commutative ring theory, Cambridge University Press, Cambridge, 1986.

[5] L. O'Carroll, On the generalized fractions of Sharp and Zakeri, J. London Math. Soc. (2), 28 (1983), 417-427.

[6] P. Schenzel, N. V. Trung and N. T. Cuong, Verallgemeinerte Cohen-MacaulayModuln, Math. Nachr., 85 (1978), 57-73.

[7] R. Y. Sharp and H. Zakeri, Modules of generalized fractions, Mathematika, 29 (1982), $32-41$.

[8] R. Y. Sharp and H. Zakeri, Local cohomology and modules of generalized fractions, Mathematika, 29 (1982), 296-306.

[9] R. Y. Sharp and H. Zakeri, Generalized fractions, Buchsbaum modules and generalized Cohen-Macaulay modules, Math. Proc. Camb. Phil. Soc., 98 (1985), 429-436.

[10] J. Stückrad and W. Vogel, Buchsbaum rings and applications, Springer Verlag, Berlin, 1986.

[11] N. V. Trung, Absolutely superficial sequences, Math. Proc. Camb. Phil. Soc., 93 (1983), 35-47.

[12] H. Zakeri, d-sequences, local cohomology modules and generalized analytic independence, Mathematika, 33 (1986), 279-284. 
K. Khashyarmanesh

Institute for Studies in Theoretical

Physics and Mathematics (IPM)

P.O. Box 19395-5746

Tehran, Iran

Sh. Salarian

Institute for Studies in Theoretical

Physics and Mathematics (IPM)

P.O. Box 19395-5746

Tehran, Iran

H. Zakeri

Institute for Studies in Theoretical

Physics and Mathematics (IPM)

P.O. Box 19395-5746

Tehran, Iran

ZAKERIH@KARUN.ipm.ac.ir 